\title{
Herpes zoster radiculopathy as a rare cause of foot drop: a case report
}

Gene Leung, MB, ChB, MRCSEd

\author{
Department of Orthopaedics and Traumatology, Pamela Youde Nethersole Eastern Hospital, Hong Kong
}

*Corresponding author: geneleung@gmail.com

Hong Kong Med J 2021;27:213-5

https://doi.org/10.12809/hkmj208465

\section{Case report}

A 69-year-old woman with a medical history of hypertension and hyperlipidaemia presented to the Medicine and Geriatrics unit of Pamela Youde Nethersole Eastern Hospital with acute onset of right leg rash for 5 days. The rash was associated with burning pain and dysesthesia along the right leg. There was no back pain and no history of back injury. She was initially diagnosed with a herpes zoster infection and treated with a course of acyclovir and amitriptyline and then discharged home. The patient presented again 10 days later for new-onset right big toe weakness with right foot drop. On the second admission, an orthopaedic consultation was sought.

Physical examination revealed a cutaneous papulovesicular rash and vesicles along the lateral aspect of her right leg down to the dorsum of her right foot (Fig 1). The rash corresponded to an L5 dermatomal distribution. Testing of sensation showed hyperesthesia along the same region. Right hip abduction (gluteus medius) power was Medical Research Council (MRC) grade 4. Right hip flexion (iliopsoas), right hip extension (gluteus maximus), right knee flexion (hamstrings), right knee extension (quadriceps) and right ankle plantar flexion (gastrocnemius) demonstrated full power of MRC grade 5. She had right foot drop with right ankle dorsiflexion (tibialis anterior tendon), MRC grade 2. Right big toe dorsiflexion (extensor hallucis longus) showed a power of MRC grade 1. Right big toe plantar flexion (flexor hallucis longus) tested MRC grade 4 power. Right ankle inversion (tibialis posterior) and right ankle eversion (peronei) elicited MRC grade 3 power. Lasègue's sign was positive for the right lower limb but there was no tenderness along the spine. There were no neurological deficits of her left lower limb. Achilles tendon and patellar tendon reflexes were normal, and sphincter function was not disturbed.

Plain radiographs of the lumbosacral spine showed mild degeneration over the lower lumbar facet joints. The pedicles and endplates were intact with no vertebral collapse or slippage. The patient was referred for magnetic resonance imaging of the lumbosacral spine to exclude concomitant compression of the neurological elements (Fig 2).

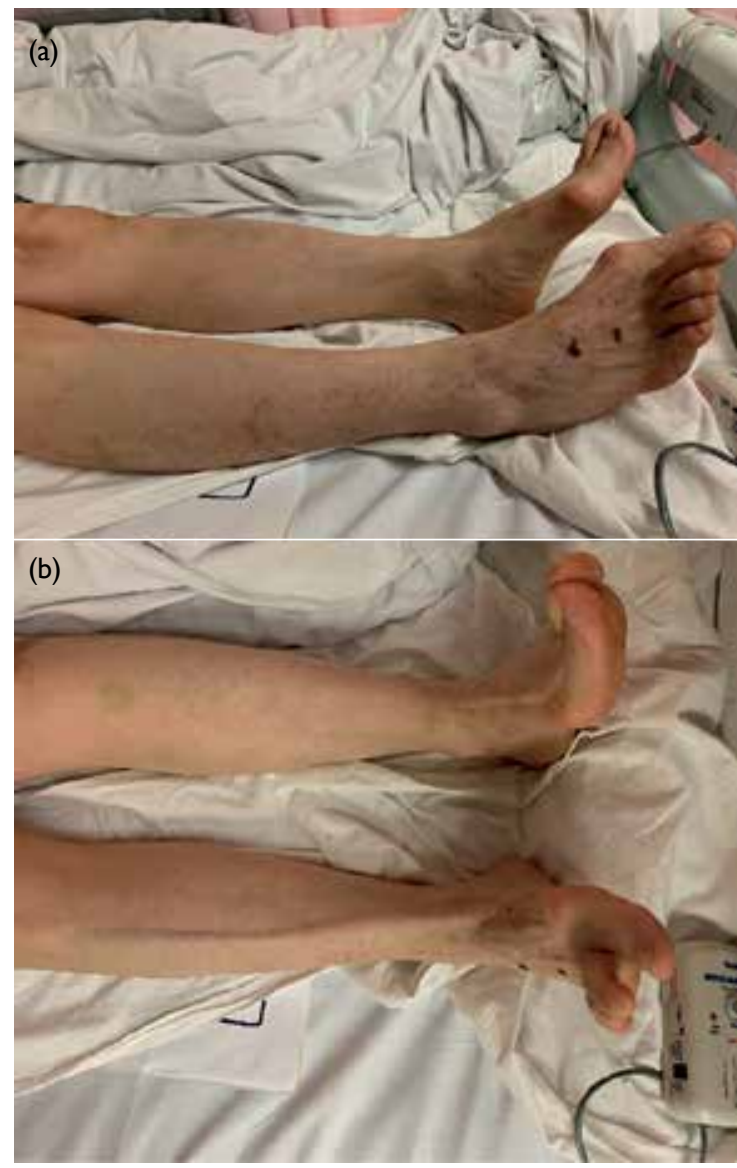

FIG I. A 69-year-old woman with acute-onset right leg rash for 5 days, initially diagnosed as herpes zoster infection. The patient presented to the hospital again 10 days later with right foot drop. Clinical photographs of the right leg on the second admission showing a vesicular rash with right L5 dermatomal distribution

There was no intervertebral disc prolapse or nerve root impingement and lateral recess and intervertebral foramen were not stenotic.

Pain medication was stepped up to gabapentin. She was discharged with a course of out-patient physiotherapy.

At 6-week follow-up examination, the vesicles had crusted, and the neuropathic pain had subsided. The power of right sided hip abduction, big toe 


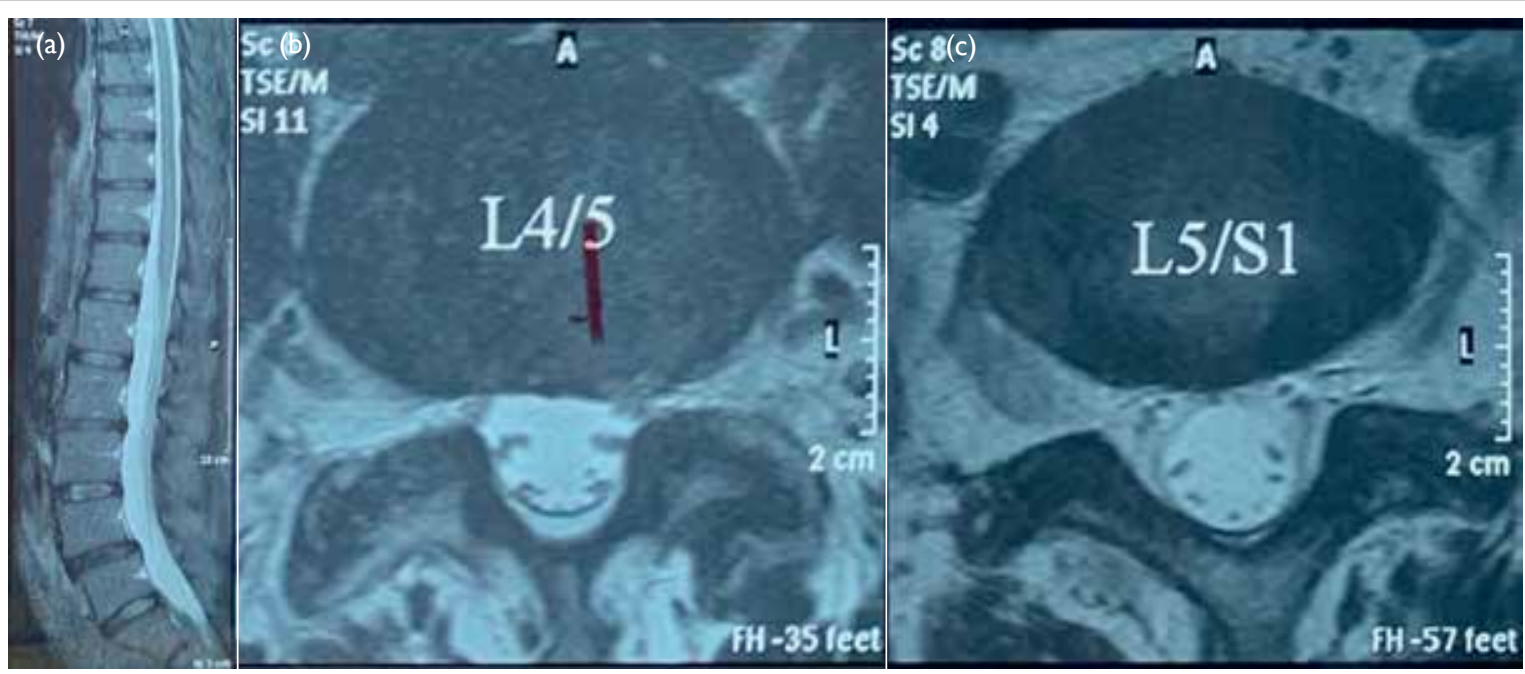

FIG 2. Same patient. (a) Sagittal magnetic resonance imaging (MRI) of the lumbar spine in T2, and axial MRI of the lumbar spine at intervertebral levels (b) $L 4 / 5$ and (c) $L 5 / S I$ show no significant disc prolapse, no spinal canal stenosis, and no foraminal or lateral recess stenosis

plantar flexion, ankle plantarflexion, ankle inversion and ankle eversion now returned to full. There was residual weakness in right ankle dorsiflexion, with a power of MRC grade 4, and right big toe dorsiflexion, with a power of MRC grade 2 .

At 3-month follow-up examination, the rashes over the right leg and foot had disappeared, leaving faint pigmentation (Fig 3). She was pain free. Right ankle dorsiflexion power was full and right big toe dorsiflexion power further improved to MRC grade 3. She was weaned off gabapentin and continued with physiotherapy for ankle strengthening exercises.

\section{Discussion}

Herpes zoster is a viral infection of the nervous system that results from the reactivation of a previous varicella zoster virus infection, often in childhood. The virus lays dormant in the dorsal root ganglion re-emerging as the patient ages or becomes immunocompromised. ${ }^{1}$ It manifests with a typical cutaneous vesicular rash that follows a spinal nerve distribution. Classically, it is believed that the virus spreads from the dorsal root ganglion in an antegrade fashion along the spinal nerve. Thus, symptoms are most often purely sensory. Rarely, when motor manifestations are present, they tend to affect more proximal muscle groups. ${ }^{2}$ The typical example is Bell's palsy due to facial nerve involvement. It has been hypothesised that local inflammation of the dorsal root ganglion results in hypervascularity in the surrounding nerve tissue, disrupting the blood-nerve barrier which can also result in a motor deficit. ${ }^{3}$ Therefore, although postherpetic neuralgia is a common manifestation of herpes zoster infection, segmental zoster paresis

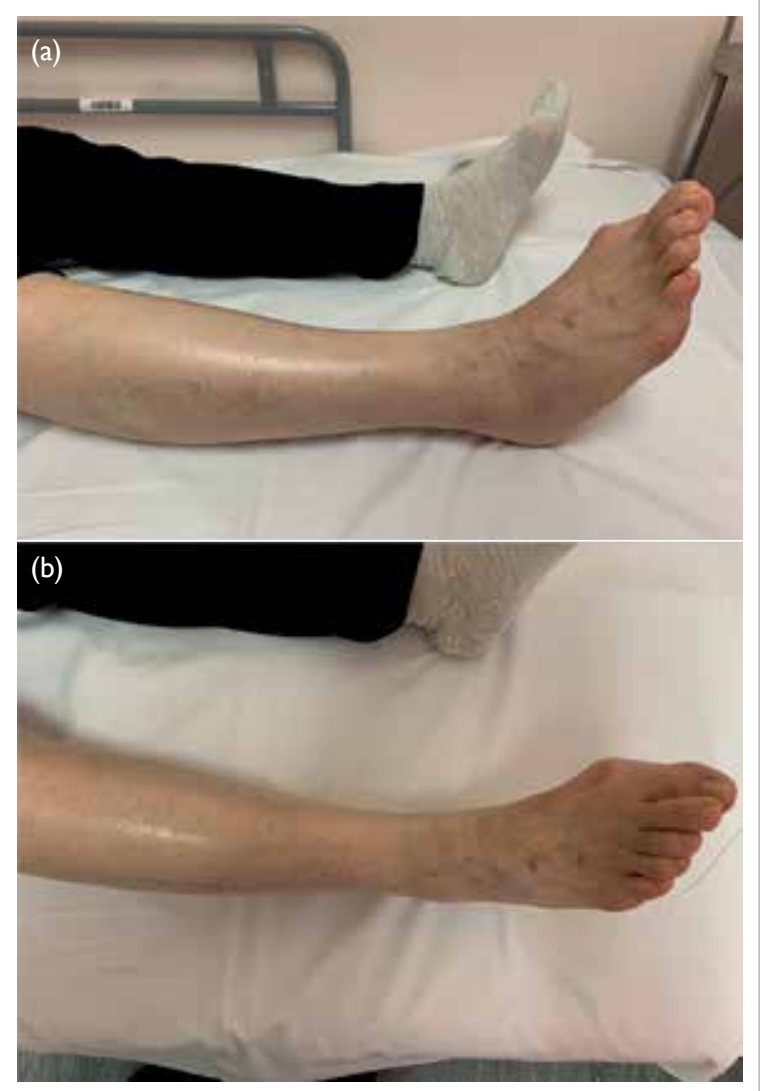

FIG 3. Same patient. Clinical photographs of the right leg at 3-month out-patient follow-up examination showing resolution of the rash with residual subtle pigmentation

is rare. Although reported incidences in different countries vary, a recent study performed with a majority of Chinese patients reported paresis in only 
$0.57 \%$ of those afflicted with a zoster infection. ${ }^{2}$ Since lumbar radiculopathy resulting from intervertebral disc herniation with nerve root irrigation commonly involves L4/5 and L5/S1 levels, a herpes zoster infection with segmental paresis affecting the L5 spinal nerve is a close mimicker. In some instances, the motor weakness can precede the rash. ${ }^{4}$

The diagnosis of herpes zoster radiculopathy is clinical although it can be aided by serum antibodies, electrodiagnostic studies and formal dermatological assessment. For this case, although electrodiagnostic testing would have been ideal to supplement the overall assessment, it was not arranged due to the anticipated recovery and resource limitations in the public hospital setting. As the lumbar spinal nerve roots were involved, the clinical presentation resembled compressive pathologies that are commonly seen in orthopaedics. A plain magnetic resonance imaging can exclude the presence of nerve root impingement, although findings that may suggest zoster infection such as spinal nerve swelling with T2 hyperintensity are neither sensitive nor specific. $^{3}$

The mainstay of treatment for zoster radiculopathy is pharmacological. A course of antivirals is prescribed for the acute herpetic infection. Pain is managed according to the World Health Organization analgesic ladder or with specific neuropathic pain medication such as amitriptyline, gabapentin or pregabalin. Although the clinical course is variable, most patients can expect to make a nearcomplete motor recovery within 1 year. ${ }^{2}$ A well-fitted ankle-foot orthosis facilitates ambulation if there is persistent foot drop. In addition, transforaminal epidural steroid injections have been reported to mitigate pain and weakness by suppressing inflammation along the spinal root. ${ }^{5}$ However, the use of corticosteroids remains controversial as there is a theoretical risk of viral reactivation. ${ }^{3}$

\section{Author contributions}

The author contributed to the concept of the study, acquisition and analysis of data, drafting of the manuscript, and critical revision for important intellectual content. The author had full access to the data, contributed to the study, approved the final version for publication, and takes responsibility for its accuracy and integrity.

\section{Conflicts of interest}

The author has disclosed no conflicts of interest.

\section{Funding/support}

This case report received no specific grant from any funding agency in the public, commercial, or not-for-profit sectors.

\section{Ethics approval}

The patient was treated in accordance with the Declaration of Helsinki. The patient provided informed consent for all procedures.

\section{References}

1. Hope-Simpson RE. The nature of herpes zoster: a long-term study and a new hypothesis. Proc R Soc Med 1965;58:9-20.

2. Liu Y, Wu BY, Ma ZS, et al. A retrospective case series of segmental zoster paresis of limbs: clinical, electrophysiological and imaging characteristics. BMC Neurol 2018;18:121.

3. Hackenberg RK, von den Driesch A, König DP. Lower back pain with sciatic disorder following L5 dermatome caused by herpes zoster infection. Orthop Rev (Pavia) 2015;7:6046.

4. Teo HK, Chawla M, Kaushik M. A rare complication of herpes zoster: segmental zoster paresis. Case Rep Med. 2016;2016:7827140.

5. Conliffe TD, Dholakia M, Broyer Z. Herpes zoster radiculopathy treated with fluoroscopically-guided selective nerve root injection. Pain Physician 2009;12:851-3. 